\title{
BRECHAS EN EL USO DE INTERNET: UN FRENTE DE ACCIÓN A LA ZAGA EN COLOMBIA
}

\section{Gaps in the use of the Internet: A front of action far behind in Colombia}

\author{
Patricia Eugenia Martínez-Coral
}

Ph.D. Universidad Externado de Colombia (Bogotá, Colombia).pattymcoral@hotmail.com

(Recibido julio 11 de 2017 y aceptado diciembre 22 de 2017)

\section{Resumen}

El presente artículo expone los resultados de una investigación de carácter exploratorio, a través de la cual se analizó el alcance de los proyectos de inversión ejecutados por el Fondo de Tecnologías de la Información y las Comunicaciones, durante los dos últimos cuatrienios, para fomentar el acceso y uso de Internet en los hogares de estratos socio-económicos bajos. Elementos tales como la cobertura, oferta de servicios, selección de beneficiarios y ejecutores, diseño de las subvenciones aplicadas, y monto de la inversión, sirvieron como criterios orientadores en el desarrollo del análisis, y fueron complementados con la reconstrucción del contexto sectorial en el que cada proyecto ha sido implementado. El estudio de la política de servicio universal en Colombia permite concluir que existe un vacío ostensible de las dimensiones de uso que deben acompañar la oferta de infraestructura. Si bien, el despliegue subsidiado de redes de última milla, y el establecimiento de tarifas máximas del servicio de banda ancha, apuntan a superar barreas de asequibilidad que obstaculizan la difusión tecnológica, resulta imperativo desarrollar estrategias que incrementen la promesa de valor de uso de Internet, de tal modo que los beneficiarios no solo retribuyan la inversión pública mediante su disposición a pagar por el servicio, fortaleciendo la sostenibilidad de este tipo de proyectos, sino que además aprovechen las condiciones de acceso haciendo uso productivo de la red.

Palabras clave: Política de servicio universal; brechas de uso; banda ancha; brecha digital.

\section{Abstract}

This article presents the results of an exploratory research, through which the scope of investment projects executed by the Universal Service Fund, during the last eight years to promote access and use of the Internet in households with low socioeconomic strata, was analyzed. Elements such as coverage, service offerings, selection of beneficiaries and executors, design of applied subsidies, and amount of investment, served as guiding criteria in the development of the analysis, and they were complemented by the reconstruction of the sectoral context in which each project has been implemented. The study of the universal service policy in Colombia allows to conclude that there is a significant gap in the dimensions of use that must accompany the supply of infrastructure. Although the subsidized deployment of last-mile networks, and the establishment of maximum broadband service tariffs, aim to overcome affordability barriers that hinder technological diffusion, it turns out to be imperative to develop strategies that increase the promise of value of Internet use, in such a way that the beneficiaries not only reward the public investment by their willingness to pay for the service, strengthening the sustainability of these types of projects, but also taking advantage of the conditions of access, by making productive use of the network.

Key words: Universal Service Policy; Usage Gaps; Broadband; Digital Divide. 


\section{INTRODUCCIÓN}

En la medida que la recolección, procesamiento y análisis de datos acerca del acceso y uso de Internet han aumentado alrededor del mundo, los Estados cuentan en la actualidad con mejores herramientas para la formulación de políticas de inclusión digital. Un primer referente de análisis está dado por la acotación de expectativas relacionadas con el aumento de penetración de Internet y sus efectos sobre la productividad y el crecimiento económico. En 2009 el Banco Mundial proyectaba que un incremento de $10 \%$ en la tasa de penetración de Internet se correlacionaba con un aumento de $1.35 \%$ en el Producto Interno Bruto de países en desarrollo, y $1.19 \%$ de países desarrollados [1]. Sin embargo, de acuerdo con los resultados obtenidos en la investigación regional desarrollada en 2014 por la red DIRSI (Diálogo Regional Sobre la Sociedad de la Información), acerca del impacto efectivo de la difusión de banda ancha en aspectos clave para el desarrollo económico en América Latina, se observa que el aumento en la penetración de banda ancha puede tener impactos positivos en la superación de pobreza, siempre que el aumento de infraestructura esté acompañado de inversión en capital humano, de modo que pueda traducirse en incrementos de productividad; de igual forma, si bien la difusión de banda ancha contribuye a la generación de nuevos empleos, simultáneamente destruye otros [2].

Un segundo ámbito de discusión, que ha adquirido notable importancia para orientar la toma de decisiones en materia de intervención pública, consiste en el estudio de las brechas de uso de Internet, a partir de las cuales es posible estimar la pertinencia y efectividad de diversas acciones tendientes a promover la difusión tecnológica, es decir, la aplicación de Internet en una diversa gama de actividades de la vida cotidiana y la producción de bienes y servicios. Según la Unión Internacional de Telecomunicaciones, en 2016 cerca de 3.9 billones de personas en el mundo continúan excluidas del acceso a Internet, cifra cercana a $53 \%$ de la población global. A su vez, mientras $80 \%$ de los ciudadanos de los países desarrollados están conectados, en los países en desarrollo este porcentaje se aproxima tan solo a la mitad (40\%); y en el grupo de países menos desarrollados, pese a que la penetración de Internet se ha triplicado en el último lustro, los niveles de acceso alcanzan aquellos registrados en los países desarrollados en 1998. De otro lado, las estadísticas de uso sugieren que las personas con mayores niveles de ingreso y escolaridad emplean los recursos de la red de manera más productiva, en tanto que aquellos con menores niveles de ingreso $y$ escolaridad usan el servicio de Internet con fines de comunicación y entretenimiento, lo que refleja el afianzamiento de dimensiones estructurales de la desigualdad [3].

En 2017, el Centro Nacional de Consultoría reveló que $43 \%$ de los colombianos se encuentra en el nivel más básico del índice de Apropiación Digital, lo cual significa que el mayor uso del servicio corresponde a entretenimiento y comunicación; $27 \%$ registra usos más avanzados, tales como interacciones de carácter comercial y educación virtual; y solo $4 \%$ se apalanca en el servicio para producir ingresos [4].

Estos hallazgos advierten sobre la necesidad de examinar cuáles son los instrumentos empleados en el marco de la política de servicio universal en Colombia, para estimular el acceso y uso de Internet, y comprobar si la inversión pública destinada para su respectiva financiación está generando los efectos propicios para superar las problemáticas de la brecha de uso, de la que se desprende una creciente modalidad de desigualdad social en el país.

\section{METOdOLOGÍA}

El presente artículo expone los resultados de una investigación de carácter exploratorio en torno a la política de servicio universal de telecomunicaciones ejecutada en Colombia por el Fondo de Tecnologías de la Información y las Comunicaciones, en adelante Fondo TIC.

Teniendo en cuenta la imposibilidad de aislar la participación de los beneficiarios de los proyectos de servicio universal, de los reportes de abonados al servicio de Internet, cualquier ejercicio estadístico sobre la incidencia de la acción pública en el comportamiento de las tasas de penetración y uso de la conectividad, conduciría a correlaciones espurias; así como tampoco se podría desconocer la estrecha relación entre la factibilidad de dichos proyectos y condiciones exógenas a la intervención del Estado.

Dificultades similares se presentan para abordar un análisis normativo de política, toda vez que sus piezas constitutivas [5] aún se encuentran en proceso de definición. Así, por ejemplo, en el estudio de la política de servicio universal en Colombia no 
resulta claro que la unidad de análisis (nacional o municipal) abarque diferencias que coincidan con la heterogeneidad regional que caracteriza al país en términos de acceso y uso de Internet; la relevancia de algunos actores, diferentes al Ministerio TIC y los beneficiarios de sus proyectos, como operadores de telecomunicaciones $y$ ente regulador, no ha sido puntualizada en la formulación de las intervenciones públicas; $y$ tampoco algunos aspectos esenciales para la delimitación de su alcance, como duración óptima de las subvenciones, y desagregación de sus costos y beneficios. En consecuencia, el estado del arte en materia de políticas de servicio universal deberá producir mayores avances antes de que pueda abordarse una investigación más amplia.

Por lo anterior, la investigación realizada se limita al análisis de los proyectos de inversión ejecutados por el Fondo TIC, durante los dos últimos cuatrienios, en el marco de la política de servicio universal. De este modo, elementos tales como la cobertura, oferta de servicios, selección de beneficiarios y ejecutores, diseño de las subvenciones aplicadas, y monto de la inversión, sirven como criterios orientadores para contrastar el abordaje institucional de las problemáticas de acceso y uso de Internet en el país, con los referentes que provee la literatura especializada sobre la brecha emergente de apropiación tecnológica.

\section{REFERENTES DE ANÁLISIS}

Desde que el incremento de la penetración y uso de Internet fueron adoptados por consenso como imperativo global de los gobiernos, corporaciones del sector privado, y sociedad civil, en el marco de la Cumbre Mundial de la Sociedad de la Información en 2003, numerosos lineamientos de acción pública han sido formulados, entre los cuales se destacan aquellos dirigidos al fomento del despliegue de infraestructura, estímulos a la demanda, y asequibilidad del servicio. Las medidas diseñadas para mejorar las condiciones de acceso a la conectividad agrupan, comúnmente, las políticas de regulación que buscan fortalecer la competencia [6]; aumentar la eficiencia en el mercado de las telecomunicaciones a través del uso compartido de infraestructura [7]; afianzar un entorno propicio para la inversión privada mediante garantías de trato no discriminatorio entre proveedores, con base en principios de neutralidad tecnológica [8]; establecer un régimen de asignación de espectro transparente y predecible para favorecer la innovación [9]; e incluir en los procesos de asignación de licencias para uso de espectro, obligaciones específicas de cobertura, como alternativa para llevar a oferta a regiones que despiertan poco interés comercial [10]. La inversión pública, acompañada de medidas regulatorias para facilitar el acceso abierto a redes de backbone y backhaul, es otra práctica tendiente a reducir las barreras a la entrada de nuevos operadores [11].

A su vez, los estímulos a la demanda del servicio de conectividad han desempeñado un rol relevante en complemento de las estrategias enunciadas [12]. Bajo el entendido que, en un ecosistema digital la infraestructura debe responder y adecuarse a las necesidades de los usuarios, habilitar el desarrollo de servicios, y facilitar el surgimiento de nuevas aplicaciones, los objetivos de difusión tecnológica se entrelazan de manera permanente, procurando que las fuerzas de oferta y demanda converjan. Es así como los programas de alfabetización y formación de talento digital en instituciones educativas se han reconocido en la esfera internacional como una prioridad para posibilitar la participación abierta en la sociedad del conocimiento, y su respectiva profundización [13]. El apoyo al emprendimiento; el comercio electrónico; la diversificación de transacciones de gobierno en línea; el fortalecimiento del régimen de protección al usuario; los protocolos de cyber-seguridad; y las estrategias de digitalización para estimular la innovación en el sector productivo también hacen parte de las acciones enfocadas a la maduración del ecosistema digital [14]. Adicionalmente, las medidas de protección de propiedad intelectual que amparan el desarrollo de las plataformas de software, aplicaciones multimedia, y contenidos digitales, se cuentan entre los incentivos indirectos para acrecentar la demanda de la conectividad [15].

En materia de asequibilidad, los instrumentos de acción pública no han contemplado intervenciones sobre precios, debido a los daños potenciales que podría traer esta medida para el desarrollo a largo plazo del mercado, en razón de eventuales distorsiones de señales y desincentivos a la inversión privada [16]. En su lugar, la reducción de impuestos, aranceles y gravámenes a las terminales, equipos y servicios de telecomunicaciones, en beneficio tanto de usuarios como operadores, ha sido acogida como mecanismo para inducir aumentos en la penetración de Internet, a través de la disminución de costos [1718]. Otro instrumento empleado para mejorar la asequibilidad del servicio de Internet ha consistido en la oferta de subsidios, entre los cuales se 
encuentran aquellos que buscan reducir los costos del despliegue de redes de última milla, la compra de terminales, y las tarifas mensuales del servicio, en favor de los hogares de escasos recursos [19].

Pese a las diferencias en las políticas de inclusión digital que pueden observarse entre países, en virtud de su nivel de ingresos, entornos regulatorios, y desarrollo del mercado de telecomunicaciones, la formulación y ejecución de planes nacionales de banda ancha se han posicionado internacionalmente como estrategia para el desarrollo conjunto de los incentivos a la oferta y demanda de bienes y servicios que integran el ecosistema digital. Dichos planes proporcionan una visión concreta de las rutas trazadas por cada gobierno, y los horizontes de tiempo establecidos para el cumplimiento de metas específicas, generando con ello un entorno proclive a la inversión y el surgimiento de nuevos modelos de colaboración entre grupos de interés [20].

Por su parte, el monitoreo constante de los avances obtenidos mediante la ejecución de estos planes ha dado origen a múltiples indicadores globales que permiten constatar la importancia del acceso y uso de Internet. Algunos ejemplos corresponden al índice de Desarrollo de las Tecnologías de la Información y la Comunicación, elaborado por la Unión Internacional de Telecomunicaciones [3], con base en la agregación de cinco indicadores de acceso (suscripciones de telefonía fija y móvil celular, ancho de banda internacional de Internet por habitante, porcentaje de hogares con computador, y porcentaje de hogares con acceso a Internet); tres indicadores de uso (porcentaje de individuos que usan Internet, suscriptores de banda ancha fija, y suscriptores de banda ancha inalámbrica); y tres indicadores de conocimientos (tasa de alfabetización de adultos, tasa bruta de matrícula en educación secundaria, y tasa bruta de matrícula en educación superior); y el Índice NRI (Networked Readiness Index), producido por el Foro Económico Mundial [21], a partir de cuatro subíndices: (1) entorno, que estima las condiciones regulatorias y de mercado que soportan el emprendimiento, la innovación, y el desarrollo de las TIC; (2) preparación, que evalúa la disponibilidad de infraestructura, la asequibilidad de los servicios, y las competencias en el uso efectivo de las TIC; (3) uso, compuesto de indicadores que reflejan la adopción de Internet por parte de individuos, empresas y gobierno; e (4) impacto, medido como innovaciones tecnológicas, y usos sociales de la conectividad, tales como la oferta de servicios ofrecidos por el estado a través de medios electrónicos.
Otras métricas similares son el índice Global de Competitividad, del Foro Económico Mundial [22], elaborado a partir de doce pilares (instituciones, infraestructura, ambiente macroeconómico, salud y educación básica, educación superior y capacitación, eficiencia del mercado de bienes, eficiencia del mercado laboral, desarrollo del mercado financiero, preparación tecnológica, tamaño del mercado, sofisticación de los negocios e innovación); y el Índice Mundial de Innovación, producido por la Organización Mundial de Propiedad Intelectual [23], con base en dos subíndices, a saber: el de recursos invertidos en innovación, que a su vez abarca cinco pilares (instituciones; capital humano e investigación; infraestructura, de la que hace parte el acceso a Internet; desarrollo de los mercados; y desarrollo empresarial), y el subíndice de resultados de innovación, en el que se encuentran la producción de conocimientos y tecnología, y la producción creativa. Por último, se encuentran referentes puntuales de las aplicaciones de la conectividad en desarrollo urbano, caso del Cities in Motion Index, construido por el IESE Business School de la Universidad de Navarra [24], a partir de diez dimensiones (gobernanza, planeación urbana, administración pública, tecnología, medio ambiente, cohesión social, alcance internacional, transporte y movilidad, capital humano, y economía); comercio electrónico (como el E-commerce Index, de la Conferencia de las Naciones Unidas sobre Comercio y Desarrollo, que evalúa la cantidad de usuarios de Internet, servidores seguros, penetración de cuentas electrónicas, y confiabilidad postal [25]; y gobierno en línea, como lo ilustra el E-government Development Index, elaborado por el Departamento de Asuntos Económicos y Sociales de las Naciones Unidas, con fundamento en tres subíndices: servicios en línea; infraestructura de telecomunicaciones; y capital humano [26].

En la medida que los ecosistemas digitales, especialmente en países desarrollados, han experimentado notables signos de maduración, el análisis acerca de la brecha digital se ha enriquecido, y se empieza a observar un mayor énfasis en las barreras de adopción tecnológica que dependen más de las facilidades de uso, que de las condiciones del acceso a Internet [27]. Algunos criterios de aproximación a las brechas de uso son el tipo de relación establecido entre el usuario y el contenido digital, lo que permite diferenciar al usuario pasivo que consume contenidos- del activo -aquel que crea contenidos y por consiguiente emplea un mayor 
ancho de banda-; la frecuencia y duración del uso de Internet; $y$ las actividades desarrolladas en línea.

De acuerdo con Van Deursen y Van Dijk [28], el grado de escolaridad, la ocupación, y el nivel de ingresos inciden en los resultados del uso de Internet. Las personas en condiciones socio-económicas más bajas, aun cuando acceden al servicio, con frecuencia y duración altas, hacen un uso más superficial de Internet, relacionado con interacción social y entretenimiento. De otro lado, los usuarios que hacen uso regular de actividades productivas en línea, con el tiempo van desarrollando habilidades que les permiten mejorar la búsqueda de información, efectuar transacciones financieras, y ampliar sus oportunidades de crecimiento profesional. Un estudio de la Organización para la Cooperación y el Desarrollo Económicos señala, con base en los resultados de las pruebas del Programa Internacional para la Evaluación de Estudiantes PISA- de 2012, que las diferencias en el uso de Internet, asociadas con actividades de aprendizaje, entre estudiantes de mayores y menores niveles de ingreso, se relacionan con otras diferencias observadas en habilidades académicas tradicionales, aun cuando la cantidad de horas de las que pueden disponer los estudiantes para acceder a Internet en la escuela sea la misma. Por ejemplo, efectuar búsquedas en línea exige capacidades de análisis y lectura crítica, así como valorar la idoneidad de las fuentes de información, y contar con dominio en el uso del computador. Por ende, las oportunidades que ofrecen Internet, y las herramientas virtuales de aprendizaje, siguen estando segmentadas en razón de desigualdades socio-económicas [29].

Por su parte, la evolución de los contenidos digitales desempeña un rol esencial en los procesos de adopción tecnológica, por ser estos la principal fuente de motivación para conectarse a Internet. Existe, además, una relación estrecha entre el desarrollo de los contenidos y la penetración de Internet. Según PwC network, en su etapa inicial, la creación de contenidos coincide con rangos de penetración entre $5 \%$ y $13 \%$. Posteriormente, los efectos de red que permiten que debido al uso común de un contenido éste ofrezca mayor valor y por ende tenga mayores posibilidades de ser usado, impulsan la apropiación de Internet, coincidiendo con incrementos en la tasa de penetración de $25 \%$ a $35 \%$. En su siguiente etapa, una base sólida de usuarios en línea fomenta la monetización de los contenidos, momento para el cual la penetración de Internet ya ha alcanzado un orden de $43 \%$ a $62 \%$; la última etapa consiste en la diversificación de contenidos por medio de nuevos servicios, y la penetración se equipara a un rango del $63 \%$ al 85 [30].

Adicionalmente, los dispositivos empleados para conectarse a Internet inciden en la clase de contenidos que son usados. Por las características de la conexión móvil, sus terminales presentan limitaciones para el uso de aquellas aplicaciones y plataformas que demandan la descarga de contenidos digitales de gran tamaño. Por consiguiente, el uso de Internet móvil resulta complementario, y no sustituto, del Internet fijo [31]. No obstante, las tendencias de penetración evidencian efectos de sustitución, lo cual es preocupante para los procesos de adopción tecnológica. La Unión Internacional de Telecomunicaciones indica que las suscripciones de Internet móvil han crecido en el mundo, en promedio, $20 \%$ cada año durante el último lustro; mientras que las suscripciones de Internet fijo han aumentado 9\% anualmente [32]. De acuerdo con el monitoreo de tráfico global de Cisco, los datos móviles han aumentado su tamaño en dieciocho veces, durante los último cinco años, alcanzando 7,2 exabytes al mes, mientras que en 2011 dicho tráfico era de 400 petabytes al mes; aunque $45 \%$ de los dispositivos móviles en el mundo corresponden a smartphones, éstos representan $81 \%$ del total del tráfico de datos. En promedio, la velocidad de las conexiones de red móvil en el mundo en 2016 fue de 6,8 Mbps. En 2021 se estima que tres cuartas partes del tráfico de datos móviles sean provistas mediante redes $4 G$, y la velocidad promedio de la conexión ascienda a $20 \mathrm{Mbps}$ [33].

Estas observaciones, en torno a las brechas de uso, comprueban la importancia de formular las iniciativas de acceso a Internet a partir de una perspectiva integral, consecuente con la necesidad de propender por la máxima efectividad posible en el logro de los cometidos de acción pública, y optimizar los recursos de inversión.

\section{RESULTADOS DEL CASO COLOMBIANO}

\subsection{Magnitud de las brechas de acceso y uso de Internet en Colombia}

El corolario expuesto contrasta con el diseño e implementación de la política de servicio universal en Colombia, contenida en los dos últimos planes nacionales de banda ancha, ejecutados desde la promulgación de la Ley 1341 de 2009: el plan vive 
digital (2010-2014), y el plan vive digital para la gente (2014-2018). Después de implementar, de manera ininterrumpida dichos planes, el gobierno colombiano ha puesto en marcha diversos incentivos a la oferta y demanda de servicios, en procura del desarrollo del ecosistema digital. En este periodo, en promedio, el Fondo TIC ha ejecutado anualmente un presupuesto de 1,2 billones de pesos $\{34\}$.

En términos de acceso a Internet se observan avances significativos. Entre 2008 y 2016, la penetración de acceso fijo creció a una tasa promedio de $5 \%$, alcanzando $16 \%$ en municipios de más de 900.000 habitantes. La velocidad promedio de conexión ha mejorado, aunque sigue estando muy por debajo de los promedios registrados en países desarrollados; en 2016 cerca de $23 \%$ de las conexiones contaba con una velocidad de $10 \mathrm{Mbps}$, $9,2 \%$ oscilaba en un rango de velocidad entre 2-5 Mbps, y $63.8 \%$ se ubicaba entre 1-2 Mbps. También se ha observado una disminución progresiva de las tarifas, aproximadamente 38\% entre 2011 y 2016; y el número de proveedores de Internet fijo ha aumentado, pasando de 33 compañías en 2011, a 87 en 2016 \{35\}.

En la actualidad, la cobertura de redes móviles en el país excede $90 \%$ en el caso de $3 G$, y la cobertura de 4G se aproxima a dos tercios de la población. GSMA Intelligence estima que el valor del sector móvil incrementará de 3.8\% del PIB en 2016, a 4.2\% del PIB proyectado en 2020 [36]. Lo anterior, pese al revés que sufrieron los incentivos tributarios, tras la reforma de 2016 [37].

Sin embargo, la caracterización de las condiciones de uso en la encuesta de hogares refleja que aún hay mucho por abordar mediante la política de inclusión digital. Según el Departamento Administrativo Nacional de Estadística \{38\}, en 2016 en Colombia $45,2 \%$ de los hogares tenía una terminal (computador, portátil, o tableta), y $45,8 \%$ una conexión a Internet (36,9\% fija; $21,8 \%$ móvil; y $12,9 \%$ fija y móvil). Entre las razones que manifestaron los hogares para no contar con una conexión a Internet, $46,3 \%$ lo considera muy costoso; $33,7 \%$ no lo considera necesario; y en orden de importancia continúa el no saber cómo usarlo $(5,7 \%)$. A su vez, $72,1 \%$ de los colombianos de cinco y más años de edad tenía un teléfono celular $(63,5 \%$ tiene un Smartphone). La proporción de personas que usó Internet desde cualquier dispositivo ascendió a $58,1 \%$, y $82,8 \%$ correspondió a personas en un rango de edad entre 12 y 24 años. Entre quienes usaron Internet, el sitio de mayor uso fue el hogar $(74,7 \%)$, seguido del trabajo $(28,1 \%)$, y la escuela $(25,3 \%)$. El dispositivo más usado para acceder a Internet, por las personas entre cinco y más años de edad, fue el celular $(70,4 \%)$, seguido del computador de escritorio $(53,2 \%)$, portátil $(30,6 \%)$ y tableta $(11,4 \%)$; y las actividades de mayor uso on-line, en las zonas urbanas donde se registran las tasas más altas de penetración, fueron entretenimiento $(76,9 \%)$, correo y mensajería $(68,7 \%)$, búsqueda de información $(67,6 \%)$, educación y aprendizaje $(39,8 \%)$, medios de comunicación $(18,4 \%)$, banca electrónica (11\%), compra de productos y servicios $(10,8 \%)$, y trámites con entidades gubernamentales $(9,1 \%)$.

\subsection{Reconstrucción de la política de servicio universal} La primera iniciativa de fomento a la penetración de Internet, en beneficio de hogares de estratos socioeconómicos bajos, fue lanzada por el Fondo TIC en 2011. Para entonces, el país registraba un número de 2.857.231 suscriptores a Internet fijo (99.2\% de los cuales contaban con servicio de banda ancha, conforme lo establecido en la Resolución CRC 2352 de 2010; esto era una velocidad efectiva de bajada (downstream) mínima igual o superior a $1.024 \mathrm{Kbps}$ y una velocidad efectiva de subida (upstream) mayor 0 igual a $512 \mathrm{Kbps})$. Cerca de $58 \%$ de los accesos dedicados fijos a Internet se concentraban en las principales ciudades del país -Bogotá D.C., Medellín, Cali, Barranquilla y Bucaramanga-, por lo cual, el proyecto buscaba promover la masificación de accesos fijos tomando como universo potencial para su ejecución un total de 325 municipios, en los que se reportaba la disponibilidad de redes troncales de fibra óptica [38].

EI Fondo TIC asignó un presupuesto de $\$ 88.485 .000 .000$ pesos (valor constante 2011) para financiar el diseño, instalación, operación, administración y mantenimiento de redes de última milla para prestar el servicio de banda ancha en hogares de estratos 1 y 2, siempre que acreditaran ser nuevos usuarios, es decir, carecer de conexión a Internet en su predio, y no haber contado con ella en el transcurso de 6 meses anteriores. Los proveedores interesados en participar en el proceso de selección objetiva estaban llamados a presentar su propuesta para los municipios potenciales de su elección, obligándose a garantizar la prestación continua del servicio durante, al menos, tres (3) años, mientras el usuario así lo decidiera. En cualquier caso, el número de accesos propuesto en la oferta debía mantenerse activo durante la ejecución del contrato, aun cuando el operador tuviera que remplazar usuarios que 
solicitaran la desconexión. Los proveedores se encontrarían en libertad de ofrecer servicios adicionales a los usuarios, y la venta de computadores, sin que su adquisición se convirtiera en requisito para acceder al acceso a Internet. El proyecto no contempló metas específicas de apropiación en el uso de Internet [39].

Por su parte, la Comisión de Regulación de Comunicaciones elaboró el estudio económico requerido para establecer el monto máximo de fomento para el despliegue de los accesos, y la tarifa mensual que el usuario debía pagar para acceder al acceso. Tomando como referente de costoeficiencia, la tecnología de redes de acceso por par de cobre (XDSL), según comparación de mercado, el regulador estimó como monto máximo de fomento por acceso $\$ 370.000$ pesos (valor constante 2011), incluido IVA y demás impuestos aplicables, señalando que este tipo de subsidio a la oferta, es recomendable por cuanto impacta la asequibilidad del servicio disminuyendo el costo de la inversión en bienes de capital, en adelante CAPEX, sin presentar riesgos para la sostenibilidad de la inversión. En virtud de este aporte, el plazo razonable para que los proveedores mantuviesen la operación del servicio se estableció en tres años, sin imponer cláusulas de permanencia a los beneficiarios, salvo que estos aceptaran servicios adicionales, o el suministro de terminales empaquetados con el plan. De otro lado, el valor máximo de la tarifa se fijó en \$20.000 pesos, ajustables con la variación del SMMLV, bajo condiciones no discriminatorias de calidad y de soporte post venta respecto a cualquier otro usuario [40].

Por último, el Fondo TIC incorporó topes a la distribución del presupuesto oficial, de acuerdo con los indicadores de penetración, empezando por municipios de 45.000 habitantes, con recursos de fomento máximo de $\$ 639.730 .000$ pesos, hasta municipios de más de un millón de habitantes, con un tope de $\$ 24.188 .010 .000$ pesos. El resultado de la adjudicación del proceso arrojó un total de 115.881 accesos, distribuidos en 77 municipios y tres ejecutores, para un presupuesto total de $\$ 17.361 .465 .000$ pesos (valor constante 2011), monto muy inferior al estimado [39].

Dos años después de la apertura de este proceso, el Fondo TIC lanzó una nueva iniciativa de masificación de accesos fijos. Para entonces, la penetración de banda ancha en el país era de 6.634 .659 suscriptores.
En esta oportunidad, se establecieron cantidades obligatorias de accesos por departamento, en las que se incluyeron 66 proyectos de vivienda de interés prioritario, para un total de 126.068 accesos. Los 28 departamentos contemplados se agruparon en regiones, y a cada una de ellas se le asignó un monto máximo de presupuesto, cuyo valor total ascendió a $\$ 100.000 .000 .000$ pesos (valor constante 2013). Las obligaciones previstas para los ejecutores incluyeron el diseño, instalación, operación, administración y mantenimiento de redes de última milla para prestar el servicio de banda ancha fija en hogares de estratos 1 y 2 (con una velocidad efectiva de bajada downstream- mínima igual o superior a $1.024 \mathrm{Kbps}$ y una velocidad efectiva de subida -upstream- mayor o igual a $512 \mathrm{Kbps})$, manteniéndose la condición de ser nuevos usuarios. La continuidad del servicio debía garantizarse por al menos dos (2) años, sin incluir cláusulas de permanencia, y sin alterar la cantidad de accesos adjudicados. Ante las dificultades por falta de pago de los usuarios, se previó que el servicio se suspendería en caso de no usarse, o estar en mora, por 3 meses consecutivos [41].

Adicionalmente, se incluyeron obligaciones de apropiación a cargo del ejecutor del proyecto, las cuales consistieron en disponer de una página WEB para ofrecer, al menos diez (10) contenidos y/o aplicaciones digitales de uso virtual, a través de las cuales se estimulara el uso cotidiano de Internet. Asimismo, al momento de efectuar la instalación del servicio, el operador debía hacer entrega al usuario, en formato digital -CD/DVD/USB-, de información complementaria, incorporando videos de la iniciativa gubernamental de uso responsable de las TIC ("En TIC confío"), y contenidos multimedia de libre selección, por parte del operador, para socializar los beneficios del uso de Internet [41].

Por su parte, la Comisión de Regulación de Comunicaciones actualizó el estudio para la asignación de los valores máximos de fomento y los topes a las tarifas mensuales del servicio. En tal sentido, encontró pertinente definir un factor ponderador por municipio para afectar el monto de subsidio al CAPEX a ser reconocido, según dos criterios: (i) a mayor Índice de Necesidades Básicas Insatisfechas (NBI) mayor asignación de recursos; y (ii) a menor población mayor asignación de recursos. De este modo, al costo por tecnología, que para el caso más eficiente es X DSL ( $\$ 348.000$ pesos), se 
sugirió reconocer entre 2 y 2.5 veces su valor en cerca del $40 \%$ de los municipios del país; 2.5 veces su valor para los proyectos de vivienda de interés prioritario, independiente del municipio en que se ubiquen, y 3.85 veces para los accesos a desplegar en San Andrés y Providencia. En complemento del subsidio al CAPEX, el regulador incluyó un subsidio sobre la tarifa mensual, dependiendo del periodo de operación del servicio a subsidiar, con el fin de fomentar la creación de un hábito de consumo en los hogares beneficiados. Este subsidio a los costos de operación -OPEX- oscilaba entre \$156.071 pesos para viviendas de interés prioritario en un horizonte de 12 meses de operación, hasta \$294.189 para dichos usuarios en un plazo de 24 meses. Con base en la máxima disponibilidad a pagar de los hogares a nivel nacional, se establecieron las siguientes tarifas máximas del servicio mensual: $\$ 6.400$ pesos para viviendas de interés prioritario; $\$ 12.400$ pesos para estrato 1; y $\$ 17.300$ para estrato 2 ; y la posibilidad de aplicar la modalidad de pre-pago estimando la distribución de las tarifas anteriores por hora $\{42\}$.

Pese a los ajustes en los valores de fomento reconocidos por el Fondo TIC, el proceso de selección objetiva solo contó con un oferente, que después de haber sido habilitado y evaluado, resultó adjudicatario de cinco (5) de las nueve (9) regiones incluidas en el proyecto, para un total de 143.000 accesos (entre obligatorios y los adicionales que hicieron parte de su oferta), distribuidos entre 617 municipios, y 20 departamentos del país, con un presupuesto de $\$ 59.865 .548 .415$ pesos (valor constante 2013). Un año más tarde, el Fondo realizó la apertura de un nuevo proceso de selección objetiva, para replicar el proyecto en los departamentos para los que no se presentaron ofertas, disponiendo de un presupuesto de $\$ 35.532 .584 .826$ pesos (valor constante 2014). No obstante, solo dos proponentes participaron del mismo, presentando ofertas para solo dos departamentos, sin resultar habilitados. En consecuencia, la entidad procedió a declarar desierta la licitación [43].

Nuevamente, hacia finales de 2015, el Fondo TIC emprendió un nuevo proyecto de masificación de accesos fijos a Internet banda ancha, al amparo de lo consagrado en el nuevo Plan Nacional de Desarrollo 2014-2018 (Ley 1753 de 2015). El alcance geográfico retomó los ocho (8) departamentos que no habían recibido ofrecimientos en los procesos anteriores, abarcando un total de 43.844 accesos, entre los cuales se relacionaban 100 proyectos de vivienda de interés prioritario. En este proceso se conservaron las mismas condiciones de selección de beneficiarios (nuevos usuarios); aspectos técnicos (accesos fijos de banda ancha con iguales velocidades mínimas de conexión, según lo establecido en la Resolución CRC 2352 de 2010, libertad para comercializar servicios adicionales sin imponer cláusulas de permanencia); apropiación del uso de Internet mediante la disposición de un portal WEB con contenidos instructivos; y los valores máximos de fomento, y tarifas mensuales al usuario definidos por el regulador en su estudio de 2013, ajustados a precios de 2015 [44].

Entre las diferencias observadas respecto de los procesos anteriores, se acortó a quince (15) meses el plazo mínimo para la prestación del servicio que debía garantizarse a los beneficiarios, y se incluyó entre las obligaciones del ejecutor, la entrega de un computador portátil a los hogares en viviendas de interés prioritario. El Fondo destinó un presupuesto oficial de \$118.469.959.552 pesos (valor constante 2015), distribuido por departamento, y adjudicó 6 departamentos entre 5 ejecutores, por un valor total de $\$ 85.292 .684 .422$ pesos equivalentes a 31.290 accesos [44]. A la fecha no se han implementado nuevos procesos de masificación de accesos.

La siguiente tabla presenta una síntesis del alcance de cada una de las iniciativas de servicio universal, implementadas por el Fondo TIC. Además de las variaciones en el tiempo de ejecución, se corrobora que el componente de uso y apropiación de Internet no ha estado sujeto al logro de metas específicas, por parte de los beneficiarios, lo que sin duda ofrece una notable oportunidad de mejora, con miras a fortalecer las estrategias encaminadas al cierre de las brechas de uso de la tecnología en Colombia. 
Tabla 1. Proyectos de masificación de accesos del Fondo TIC (2011-2017)

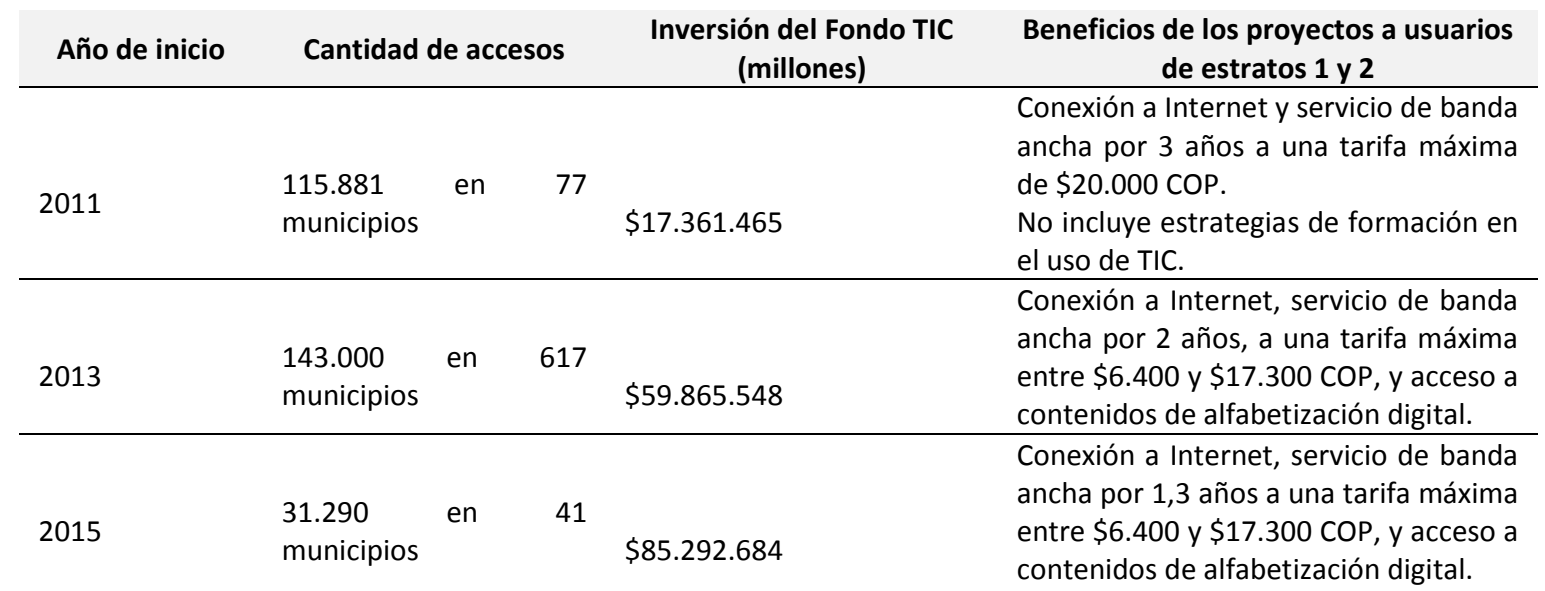

\section{CONCLUSIONES}

La reconstrucción de las iniciativas enmarcadas en la política de servicio universal en Colombia, evidencia un vacío ostensible de las dimensiones de uso que deben acompañar la oferta de infraestructura. Si bien, el despliegue de redes de última milla, bajo el esquema de subsidios al CAPEX, y el establecimiento de tarifas máximas del servicio, apuntan a superar barreas de asequibilidad que obstaculizan la difusión tecnológica, resulta imperativo desarrollar estrategias que incrementen la promesa de valor de uso de Internet, de tal modo que los beneficiarios no solo retribuyan la inversión pública mediante su disposición a pagar por el servicio, fortaleciendo la sostenibilidad de este tipo de proyectos, sino que además aprovechen las condiciones de acceso haciendo uso productivo de la red.

De esta forma, la política de servicio universal podría traducirse en el cierre de brechas, tanto de acceso como de uso, con mayores niveles de efectividad. En consecuencia, es preciso que los subsidios aplicados estén condicionados a logros específicos de formación o desarrollo de competencias por parte de los usuarios, sin que necesariamente éstos incurran en sus costos.

Entre las líneas de investigación llamadas a complementar este abordaje preliminar, se destacan la profundización del análisis sobre la estructura de mercado de telecomunicaciones en Colombia, tendiente a explicar la baja concurrencia de proveedores de redes en los procesos de selección adelantados por el Fondo TIC para promover la masificación de accesos a Internet; y los efectos potenciales que tendrá sobre la política de servicio universal, el cambio regulatorio en la definición de banda ancha, que entrará a regir a partir del 10 de enero de 2019, teniendo en cuenta que la velocidad mínima de bajada pasa a $25 \mathrm{Mbps}$, y que en este nuevo marco regulatorio no se exceptúan de las disposiciones comerciales, los proyectos de telecomunicaciones sociales [45].

\section{REFERENCIAS}

[1]C.Z. Quiang \& C.M. Rissotto, "Economic impacts of broadband". In Information and Communications for Development 2009: Extending reach and increasing impact. pp. 35-50, 2009. Washington D.C.: World Bank.

Disponible: http://documents.worldbank.org/curated/en/64582 1468337815208/071652160_201407217023713/add itional/487910PUB0EPI11010fficialOUse0Only1.pdf [2]H. Galperin, J. Mariscal, \& R. Barrantes, "The Internet and poverty: opening the black box". Lima: DIRSI, 2014.2 Disponible: https://dirsi.net/web/files/files/Opening_the_Black_ Box.pdf

[3] International Telecommunications Union, "Measuring the Information Society Report 2016". (En línea). Disponible en http://www.itu.int/en/ITUD/Statistics/Documents/publications/misr2016/MISR 2016-w4.pdf

[4] C. Lemoine, F. Alarcón, \& P. Lemoine, "Transformación y apropiación digital". Bogotá: Centro Nacional de Consultoría, 2017. 
[5] C. Robert, \& R. Zeckhauser, "The Methodology of Normative Policy Analysis". Journal of Policy Analysis and Management, Vol. 30 (3), Summer 2011, Pp. 613-643.

Disponible: https://sites.hks.harvard.edu/fs/rzeckhau/RobertZeckhauser.pdf. DOI: https://doi.org/10.1002/pam.20578

[6] H. Gruber, \& P. Koutroumpis, "Competition enhancing regulation and diffusion of innovation: the case of broadband networks", Journal of Regulatory Economics, Vol. 43 (2). pp. 168-195, 2013.

DOI: https://doi.org/10.1007/s11149-012-9205-4

[7] C. Bouras, K. Antonis, G. Diles, V. Kokkinos, \& L. Loukopoulos, "Economic Broadband Development through Infrastructure Sharing", International Journal of Business Data Communications and Networking, Vol. 10 (4), Pp. 21-42, October-December 2014. Disponible:

http://ru6.cti.gr/ru6/system/files/publications/Econo mic-Broadband-Development-through-

Infrastructure-Sharing.pdf

[8] T. Reynolds, \& S. Wunsch-Vincent, "Broadband Growth and Policies in OECD Countries, main findings". OECD, 2008. \{En línea\}. Disponible: http://www.oecd.org/internet/broadband/40629032 .pdf

[9] C. Koboldt, "Competencia y reglamentación en el mundo de la banda ancha y la convergencia", ITU: 2013. \{En línea\}. Disponible: http://www.itu.int/ITUD/treg/publications/SMP-S.pdf

[10] V. Mulas, "Policies for Mobile Broadband", In Information and Communications for Development 2012: Maximizing Mobile, world bank. Washington D.C.: The World Bank Group. 2012. Pp 103-112. Disponible:

http://siteresources.worldbank.org/EXTINFORMATIO NANDCOMMUNICATIONANDTECHNOLOGIES/Resour ces/IC4D-2012-Chapter-7.pdf

[11] OECD, "Broadband Networks and Open Access", OECD Digital Economy Papers, No. 218, Paris: OECD, 2013.DOI: https://doi.org/10.1787/5k49qgz7crmr-en [12] S. Levin, S. Schmidt, \& S. Graham, "Broadband Adoption: Translating the Digital Divide Literature into Effective Government Policies and Actions" In 27th European Regional Conference of the International Telecommunications Society (ITS), Cambridge, United Kingdom, 7th - 9th September 2016. $\quad$ En línea\}. Disponible: https://www.econstor.eu/bitstream/10419/148684/ 1/Levin-et-al.pdf

[13] UNESCO. "Digital literacy in education, Policy brief. Unesco, 2011. $\{$ En línea\}. Disponible:

Revista Ingeniería, Investigación y Desarrollo, Vol. 18 (1), 34-45, Ene. 2018. http://unesdoc.unesco.org/images/0021/002144/21 4485e.pdf

[14] World Economic Forum, "Delivering digital infrastructure. Advancing the Internet economy". WEF, 2014. $\quad$ En línea\}. Disponible: http://www3.weforum.org/docs/WEF_TC_Delivering DigitalInfrastructure_InternetEconomy_Report_2014 .pdf

[15] UNCTAD, "Internet broadband for an inclusive digital society", Current studies on science, technology, and innovation, No 11. New York, 2015. Disponible:

http://unctad.org/en/PublicationsLibrary/dtlstict201 3d4_en.pdf

[16] J. Holmes \& M. O'Rourke, "Regulating Broadband Prices". Report for the Regulatory and Market Environment Division (RME) of the Telecommunication Development Bureau (BDT). ITU, 2012. \{En línea\}. Disponible: https://www.itu.int/ITUD/treg/broadband/ITU-BB-

Reports_RegulatingPrices.pdf

[17] Broadband Commission for Sustainable Developmen, "The State of Broadband: Broadband catalyzing sustainable development September 2016". ITU, 2016.2 Disponible: http://www.broadbandcommission.org/Documents/ reports/bb-annualreport2016.pdf

[18] R. Katz, E. Flores, \& J. Mariscal, "The Impact of Taxation on the Development of the Mobile Broadband Sector". GSMA, 2012. \{En línea\}. Disponible:

https://www.gsma.com/latinamerica/wpcontent/uploads/2012/06/tasreport.pdf

[19] C. Rhinesmith, "Digital inclusión and meaningful broadband adoption initiatives". Evanston, IL: Benton Foundation, January 2016. \{En línea\}. Disponibe: https://www.benton.org/sites/default/files/broadba ndinclusion.pdf

[20] P. Biggs, "Why National Broadband Plans Matter", ITU Report Planning For Progress. Geneva: 2013. $\quad$ En línea\}. Disponible: http://www.broadbandcommission.org/documents/r eportNBP2013.pdf

[21] World Economic Forum, "Global Information Technology Report 2016". \{En línea\}. Disponible: http://reports.weforum.org/global-informationtechnology-report-2016/networked-readiness-index/ [22] World Economic Forum, "The Global Competitiveness Index 2017". \{En línea\}. Disponible: http://reports.weforum.org/global-competitivenessindex-2017-2018/\#topic=about

[23] Organización Mundial de Propiedad Intelectual (OMPI), “Índice Mundial de Innovación 2017". \{En 
línea\}.

Disponible:

http://www.wipo.int/publications/es/details.jsp?id= 4193

[24] IESE Business School Universidad de Navarra. "Cities in motion index, 2017". \{En línea\}. Disponible: http://citiesinmotion.iese.edu/indicecim/?lang=en [25] UNCTAD, “E-commerce Index, 2017". \{En línea\}. Disponible:

http://unctad.org/en/PublicationsLibrary/tn_unctad _ict4d09_en.pdf

[26] UNDESA, "United Nations E-government survey, 2016". $\quad$ En línea\}. Disponible: https://publicadministration.un.org/egovkb/enus/Reports/UN-E-Government-Survey-2016

[27] C. Davidson, M. Santorelli, \& T. Kamber, "Toward an Inclusive Measure of Broadband Adoption". International Journal of Communication, Vol. 6 (2012), Pp. 2555-2575.

[28] Van Deursen, A. \& Van Dijk, J. "The digital divide shifts to differences in usage", New media \& society 0(0), pp.1-20. \{En línea\}. Disponible: http://journals.sagepub.com/doi/abs/10.1177/1461 444813487959.

[29] OECD, "Students, Computers and Learning: Making the Connection", Paris: OECD Publishing, 2015. \{En línea\}.

DOI: https://doi.org/10.1787/9789264239555-en

[30] El-Darwiche, B., Herzog, M., Singh, M. \& Maalouf, R. "Understanding digital content and services ecosystems: The role of content and services in boosting Internet adoption", New York: PwC network, 2016. $\quad\{E n \quad l i ́ n e a\} . \quad$ Disponible: https://www.strategyand.pwc.com/reports/digitalcontent-services-ecosystems

[31] R. Katz, \& T. Berry, "Driving Demand for Broadband Networks and Services", Signals and Communication Technology, DOI: 10.1007/978-3319-07197-8_2, Springer International Publishing Switzerland, 2014.

DOI: https://doi.org/10.1007/978-3-319-07197-8_2

[32] International Telecommunications Union, "Figures and Facts, 2017". \{En línea\}. Disponible: http://www.itu.int/en/ITU-

D/Statistics/Documents/facts/ICTFactsFigures2017.p df

[33] Cisco, "Visual Networking Index: Global Mobile Data". $\quad$ En $\quad$ línea $\}$ Disponible: https://www.cisco.com/c/en/us/solutions/collateral/ service-provider/visual-networking-indexvni/mobile-white-paper-c11-520862.pdf

[34] Fondo TIC. Presupuesto anual -series- Bogotá, 2017. $\{$ En línea\}. Disponible: http://www.mintic.gov.co/portal/604/w3propertyvalue-545.html

[35] Comisión de Regulación de Comunicaciones, "Revisión del mercado de datos fijo". Bogotá, Colombia: CRC, 2017. \{En línea\}. Disponible: https://www.crcom.gov.co/recursos_user/2017/acti vidades_regulatorias/mercados/170517_mercados_ datos_fijos.pdf

[36] GSMA Intelligence, "Country overview: Colombia Mobile industry collaborating with government to promote entrepreneurship and innovation" Report 2017. \{En línea\}. Disponible: https://www.gsmaintelligence.com/research/?file=5 9bfccd5a508f91be5dabb92a6b81621\&download [37] F. Buitrago, \& C. Romero, "Consecuencias económicas de las propuestas en torno a las tic por parte de la reforma tributaria", Bogotá: CCIT, 2016. \{En línea\}. Disponible: https://www.ccit.org.co/wpcontent/uploads/consecuencias-economicas-del-plde-reforma-tributaria-2016.pdf

[38] Departamento Administrativo Nacional de Estadística, "Indicadores básicos de tenencia y uso de Tecnologías de la información y la comunicación - TIC en hogares y personas 2016", Bogotá, DANE. \{En línea\},

Disponible: http://www.dane.gov.co/files/investigaciones/boleti nes/tic/prese_tic_hogares_2016.pdf

[39] Sistema Electrónico de Contratación Pública. "Consulta de proceso -Hogares Digitales- Fondo TIC, 2011". $\quad$ En línea\}. Disponible: https://www.contratos.gov.co/consultas/detalleProc eso.do?numConstancia=11-1-73122

[40] Comisión de Regulación de Comunicaciones, "Apoyo técnico al Ministerio de Tecnologías de la Información y las Comunicaciones en la implementación de subsidios para la promoción al acceso a Internet - Ley 1450 de 2011-“, \{En línea\}. Disponible:

https://www.contratos.gov.co/consultas/detalleProc eso.do?numConstancia=11-1-73122

[41] Sistema Electrónico de Contratación Pública. "Consulta de proceso -Conexiones Digitales- Fondo TIC, 2013". $\quad$ En línea\}. Disponible: https://www.contratos.gov.co/consultas/detalleProc eso.do?numConstancia=13-1-104466

[42] Comisión de Regulación de Comunicaciones, "Esquema para la Implementación de subsidios e incentivos para el acceso a Internet de última milla". \{En línea\}. Disponible:

https://www.contratos.gov.co/consultas/detalleProc eso.do?numConstancia=13-1-104466 
[43] Sistema Electrónico de Contratación Pública. "Consulta de proceso -Conexiones Digitales fase 2Fondo TIC, 2014". \{En línea\}. Disponible: https://www.contratos.gov.co/consultas/detalleProc eso.do?numConstancia=14-1-126033

[44] Sistema Electrónico de Contratación Pública. "Consulta de proceso -Conexiones Digitales fase 2Fondo TIC, 2015". \{En línea\}. Disponible: https://www.contratos.gov.co/consultas/detalleProc eso.do?numConstancia $=15-1-139849$

[45] Comisión de Regulación de Comunicaciones, “Resolución 5161 de 2017, por la cual se establecen las definiciones y condiciones regulatorias de banda ancha en el país, y se dictan otras disposiciones". \{En línea\}. Disponible: https://www.crcom.gov.co/resoluciones/00005161.p df. 